\title{
The role of magnetic resonance imaging in acute abdominal pain in paediatric age group
}

\author{
Vijinder Arora* (1), Tanveen Kaur and Kunwarpal Singh
}

\begin{abstract}
Background: The purpose of this study is to evaluate the utility of magnetic resonance imaging in the assessment of acute atraumatic abdominal pain as the first line cross sectional modality in children, so as to prevent excessive radiation exposure from computed tomography scan and to review the magnetic resonance imaging features of common acute abdominal and pelvic conditions.

Results: 30 patients (0-18 years) underwent rapid unenhanced magnetic resonance imaging. The results of our study indicated that for the diagnosis of causes for acute abdominal pain, magnetic resonance imaging had sensitivity of $92 \%$ (95\% confidence interval $73.8-97.6 \%$ ) and a specificity of $80 \%$ (95\% confidence interval $28.4-99.5 \%)$. The positive predictive value was 95.8\% (95\% confidence interval 79.9-99.3\%) and negative predictive value was 67\% (95\% confidence interval 33.1-89.0\%) which had a highly significant statistical association $(p<0.001)$.

Conclusion: Unenhanced magnetic resonance imaging is an excellent option for the initial, detailed evaluation of acute abdominal emergencies in pediatric patients because it can diagnose the whole range of presenting abnormalities which include the causes of abdominal pain warranting surgical and nonsurgical management.
\end{abstract}

Keywords: Magnetic resonance imaging, Acute abdominal pain, Pediatric, Ovarian torsion, Acute pancreatitis, Appendicitis

\section{Background}

Acute abdominal pain is generally defined as pain of non-traumatic origin with maximum duration of 5 days. Acute atraumatic emergent abdominal pain is a challenging presentation in pediatric patients, having many potential underlying causes [1]. The diagnosis and management of these patients is further complicated by variable patient cooperation [2]. Depending on the severity of presentation and suspicion for complications, ultrasound is currently recommended for initial assessment [3, 4], although more advanced imaging may be considered for definitive assessment. CT is a highly accurate and effective cross-sectional imaging modality that is widely accessible and not user dependent [5]. There is, however, an increased awareness of the long-term risks of radiation

*Correspondence: drvijinderarora338@gmail.com

Department of Radiodiagnosis and Imaging, Sri Guru Ramdas Institute of Medical Sciences and Research, Vallah, Amritsar, Punjab 143001, India exposure associated with medical imaging. The pediatric population is at greater risk because they are inherently more radio-sensitive and have a longer remaining life expectancy during which a radiation-induced cancer may develop. This concern has prompted a search for alternatives to $\mathrm{CT}$, despite its efficacy as a diagnostic imaging tool, to minimize the risk of increasing radiation induced cancers in pediatric patients [6]. MRI as a first-line imaging protocol is being used in many clinical settings [7, 8]. A specialized MRI protocol is often used for these patients, including MRCP for the evaluation of biliary abnormalities, MR enterography for the setting of acute flare-up of inflammatory bowel disease, and a pelvic protocol for suspected cases of ovarian torsion. Regarding cross-sectional imaging performed in the setting of acute atraumatic emergent abdominal pain, multiple prior studies and meta-analysis comparing unenhanced MRI versus $\mathrm{CT}$ for the detection of causes of acute abdominal pain revealed similar excellent diagnostic performance, 
with MRI having the obvious advantage of not requiring ionizing radiation $[9,10]$.

The study aimed at exploring the role of the magnetic resonance imaging in acute abdominal pain in paediatric age group and later comparing MRI findings with surgi$\mathrm{cal} / \mathrm{lab}$ findings and evaluating sensitivity and specificity of MRI in acute abdominal pain in pediatric age group.

\section{Methods}

A prospective, single-centred, observational study was conducted on 30 patients of age group of $0-18$ years who presented to paediatric emergency department with acute atraumatic abdominal pain between September 2019 to January 2021, after obtaining approval from the institutional ethics committee/review board. All MRI scans were done on 1.5 Tesla Philips Achieva dstream. MRI findings were correlated with surgical/lab findings. The rapid protocol was designed exclusively for these emergency department patients, and none of the patients included in this review were scheduled in advance for the MRI examination. None of the patients was sedated for the MRI examination. Free breathing was used to eliminate the need to perform breath-holds. The pediatric care coordinator or a parent was permitted to be in the MRI scanner magnet room (zone IV) with the patient. No contrast agent was given. The patients were then imaged with T2 HASTE (Half Fourier Acquisition Single-shot Turbo spin Echo) sequence in axial and coronal planes. HASTE T2W sequence in sagital plane wherever it was required. Sagittal T2-weighted sequences with and without SPAIR (Spectral Attenuated Inversion Recovery) fat suppression were acquired through the pelvis. Whenever required single shot breathhold MRCP was also taken. Axial DWI was performed, and apparent diffusion coefficient maps were generated wherever required. To keep the scan duration as short as possible for a large range of patient sizes, the FOV was varied among patients. TR values were varied among patients and kept short to minimize motion and duration. Coronal acquisition parameters were as follows: median TR/TE, 2516/70; slice thickness $4 \mathrm{~mm}$; FOV, 300-400 mm; and median scan duration, $81 \mathrm{~s}$. Axial acquisition parameters were as follows: median TR/TE, 1721/110; slice thickness, $5 \mathrm{~mm}$; FOV, $300-400 \mathrm{~mm}$; and median scan duration, $77 \mathrm{~s}$. A T2-weighted axial sequence without fat saturation was also acquired with the following parameters: median TR/TE, 1832/50-90; slice thickness, $5 \mathrm{~mm}$; FOV, 300$400 \mathrm{~mm}$. The total scan duration for these sessions varied according to patient size, from $2 \min 29 \mathrm{~s}$ to $8 \min 45 \mathrm{~s}$. The MRI diagnosis later compared with the corresponding lab results and surgical results (in cases where surgery was performed).

\section{Statistical analysis}

A two-way contingency table approach was used to determine the sensitivity and specificity of the diagnosis of causes of acute abdominal pain on MRI along with negative and positive predictive values. Statistical assessment was carried out using SPSS ${ }^{\circledR} 24.0$ version $\left(\right.$ IBM $^{\circledR}$, Armonk, New York, USA). A probability threshold of 0.05 was considered as significant.

\section{Results}

Out of 30 patients, 14 (46.7\%) were girls, 16 (53.3\%) were boys, and their ages ranged from 0 to 18 years (with a mean age of 12.7 years). Amongst the cases operated for acute abdomen, there were 3 cases of appendicitis (10\%), 3 cases of ovarian torsion (10\%), 2 cases of Cholelithiasis (6.7\%), 2 cases of Intestinal obstruction (6.7\%), 1 case of intussusception, 1 of case Testicular torsion (3\%), 1 case of Meckel's diverticulum (3\%) and 1 case of small gut duplication cyst (3\%).The cases those were on follow up included acute 4 patients of pancreatitis (16\%), 1 case of urolithiasis (3\%), 2 cases of haemorrhagic cyst (6\%), 2 cases of Pelvi-ureteric obstruction (6.7\%), 1 case of tuboovarian abscess (3\%), 1 patient of hematometra (3\%) and 5 patients had no significant findings on MRI and considered to be normal (16\%). One patient had MRI finding of thickening of ileocecal junction, but on follow up, all the investigations came out to be normal. So MRI false positively detected the features of gastrointestinal tuberculosis. One case which came out to be false negative was meckel's diverticulum, which was not picked on MRI, but was found on surgery as meckel's diverticulum.

These results indicated that MRI had sensitivity of $92 \%$ (95\% CI $73.8-97.6 \%)$ and a specificity of $80 \%$ (95\% CI 28.4-99.5\%). For the MRI diagnosis of causes for acute abdominal pain, the negative predictive value was $67 \%$ (95\% CI 33.1-89.0\%) and the positive predictive value was $95.8 \%$ (95\% CI 79.9-99.3\%) with p value less than 0.001 .

\section{Discussion}

Acute abdominal pain is defined as pain in or over the abdominal cavity experienced for anything between few hours and a few weeks [4]. The time interval is necessarily blurred but majority of patients present between six and eight hours after developing pain. Although ultrasound remains the primary modality for the diagnosis of causes of acute abdominal pain in paediatric patients, it has potential drawbacks including operator dependence, poor image quality in obese children, which is becoming a significant problem [11, 12]. Given that there may be cases in which ultrasound is nondiagnostic or difficult to perform, the development of an alternative approach to $\mathrm{CT}$, the usual next imaging choice, is warranted because 
of the growing awareness of the negative effects of radiation exposure associated with medical imaging in paediatric patients. The IR(MER) regulations of 2000 declare that the justifying practitioner must assess the risks and benefits of other techniques involving less exposure [13]. It is therefore the obligation for radiologists to consider $\mathrm{MR}$ as an alternative to $\mathrm{CT}$ in cases of acute abdominal pain, particularly in paediatric patients. Our study investigated the feasibility of rapid unenhanced abdominal and pelvic MRI in the evaluation of children with acute abdominal pain and compared these findings with surgi$\mathrm{cal} / \mathrm{lab}$ findings and evaluated sensitivity and specificity of MRI in acute abdominal pain in paediatric age group.

Acute appendicitis is the most common cause of acute abdominal pain and the most common surgical condition encountered in paediatric age group. In our study, appendicitis was identified in all the 3 children who presented with pain in right iliac fossa and showed rebound tenderness. Appendicitis was seen as an enlarged appendix with appendiceal diameter greater than $7 \mathrm{~mm}$ with a thickened and oedematous wall (Fig. 1) with adjacent inflammatory changes which were best seen on T2-weighted images with fat suppression. MRI offers superior contrast resolution relative to any other available diagnostic modality. As a result detection of even small amounts of fluid and mild inflammation is possible even without the use of intravenous contrast material. Periappendiceal inflammation is a hallmark of acute appendicitis and is readily detectable on the T2-weighted fluid sensitive sequences as high signal in the soft tissues adjacent to the appendix. In one of the children who presented with acute pain in right iliac region, there were features suggesting appendicular perforation. Axial SS-TSE image demonstrated a focal walled off fluid collection with adjacent inflammatory changes (Fig. 2). Cross-sectional imaging has the benefit of detecting the appendix in cases when abnormally positioned, such as in the left abdomen in a patient with malrotation or pelvic positioning of appendix. This

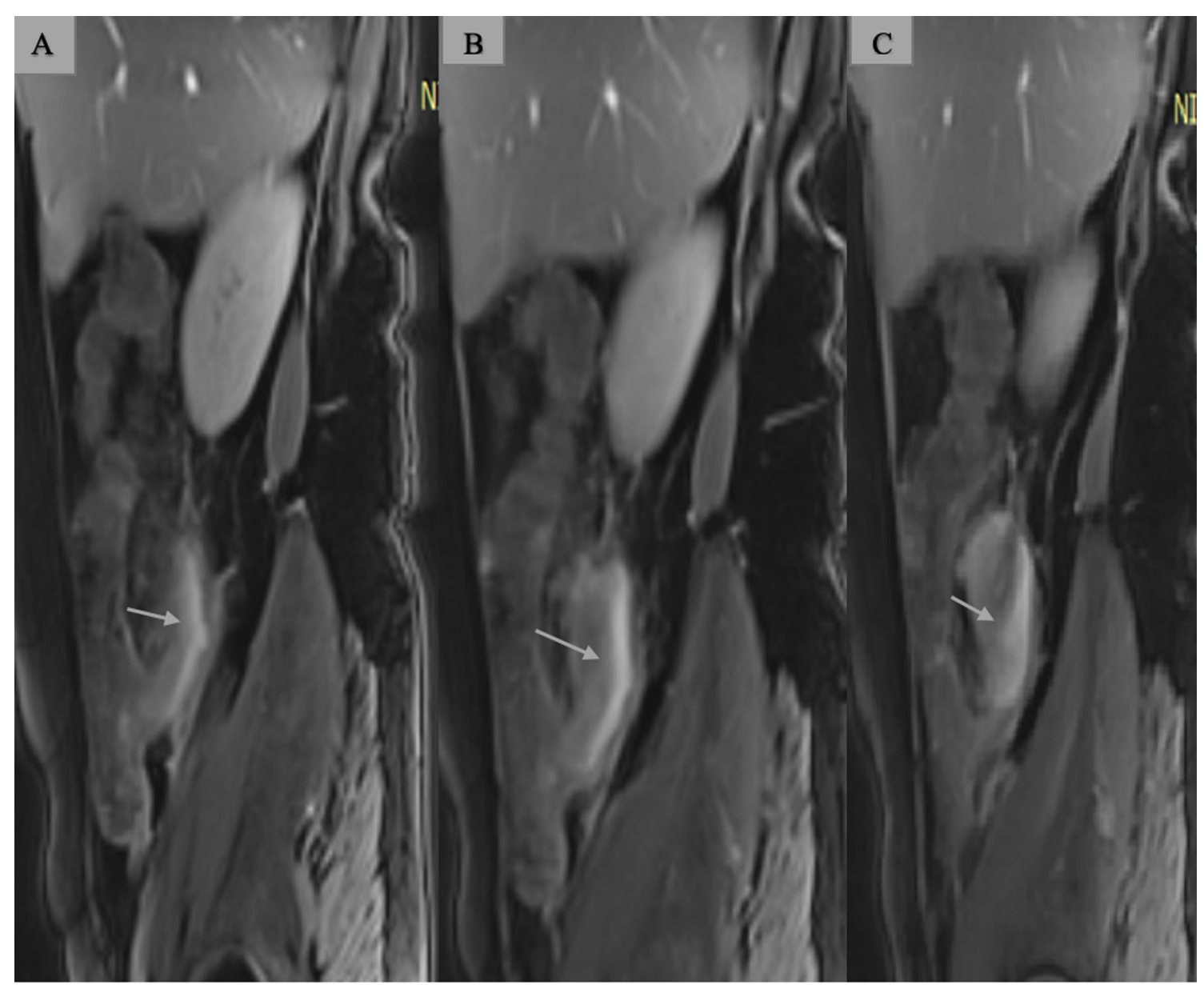

Fig. 1 T2 FS sagital MR image at different levels $(\mathbf{A}-\mathbf{C})$ showing enlarged appendix with appendiceal diameter greater than $7 \mathrm{~mm}$ with a thickened and oedematous wall (arrows). Periappendiceal inflammation can be seen in the images 


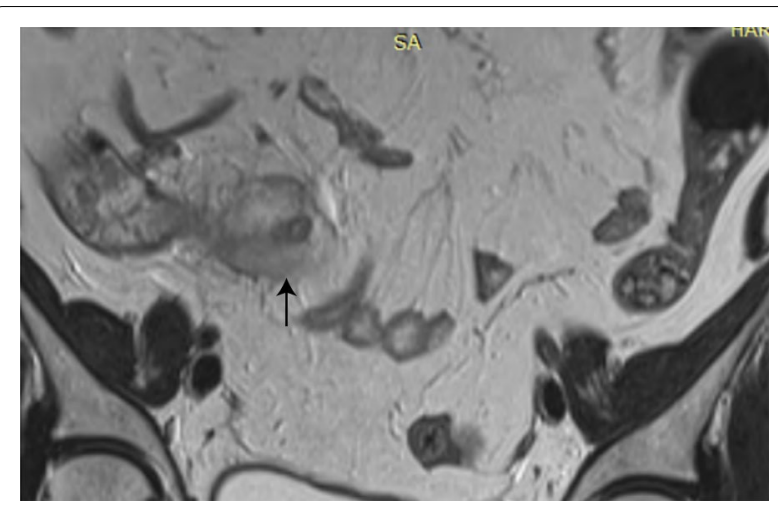

Fig. 2 T2 weighted MR image showing hyperintense collection in right iliac region-appendicular perforation

is in accordance with the results of Johnson et al. [14] who proved that Ultrafast 3-T MRI is a feasible alternative imaging modality for the diagnosis of acute appendicitis in children, in cases where ultrasound is equivocal or nondiagnostic. The normal appendix on MRI is visible as a blind-ending tubular structure arising from the cecum. The normal appendix may be filled with air (low T2 signal in an nondependent position) or fluid. Inherent non-visualization of the appendix due to adjacent bowel or lack of intraperitoneal fat implies that the appendix is not inflamed or enlarged. Essentially, an inflamed appendix will almost certainly be visible on MRI.
6 patients presented with pain in inguinal region which was acute in onset. Ovarian torsion was identified in 3 of them. Ovarian torsion results from twisting of the ovary and its vascular pedicle on its ligamentous support, and it can result in ischemia or infarction with permanent adverse effects on fertility. Early diagnosis and surgical intervention are critical in salvaging ovarian function. However, the clinical findings in children may be nonspecific, mimicking more common intestinal or urologic abnormalities, and CT or MRI is used as the initial imaging study $[15,16]$. In addition, MRI may be requested as a next step in cases where there is incomplete characterization of the ovaries on ultrasound, CT, or both. In our study three MRI findings of ovarian torsion included enlarged, edematous ovary, twisted pedicle, and ovarian hemorrhage (Fig. 3). A torsed ovary showed central edema and prominent peripheral cysts, which were best seen on a T2-weighted sequence through the pelvis. Sintim-Damoa et al. [16] conducted study in which it was suggested that ovarian torsion findings are best seen on T2 weighted images with fat suppression. In 2 of these patients MRI findings suggested the features of hemorrhagic cyst which on follow up proved to be the same (Fig. 4).

1 of these patient, who presented with acute inguinal pain which was periodical showed the features of hematometra on MRI (Fig. 5) and 1 patient showed the features of tubo-ovarian abscess (Fig. 6). The axial T2WI(A) in this patient showed encapsulated hyperintense contents,
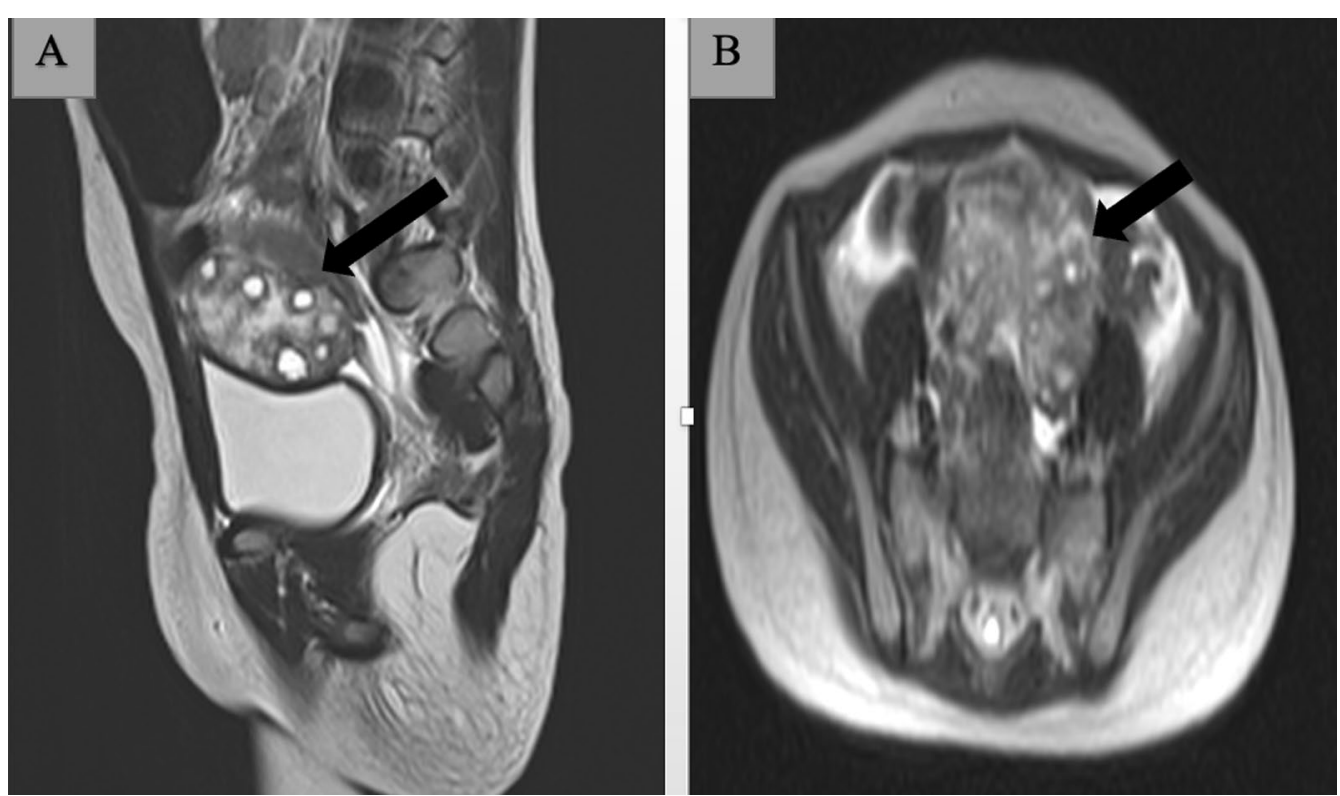

Fig. 3 Sagital (A) and axial (B) T2 weighted MR image shows an ovarian enlargement with a central afollicular region secondary to hemorrhage and edema (arrow), as well as peripherally located follicles 

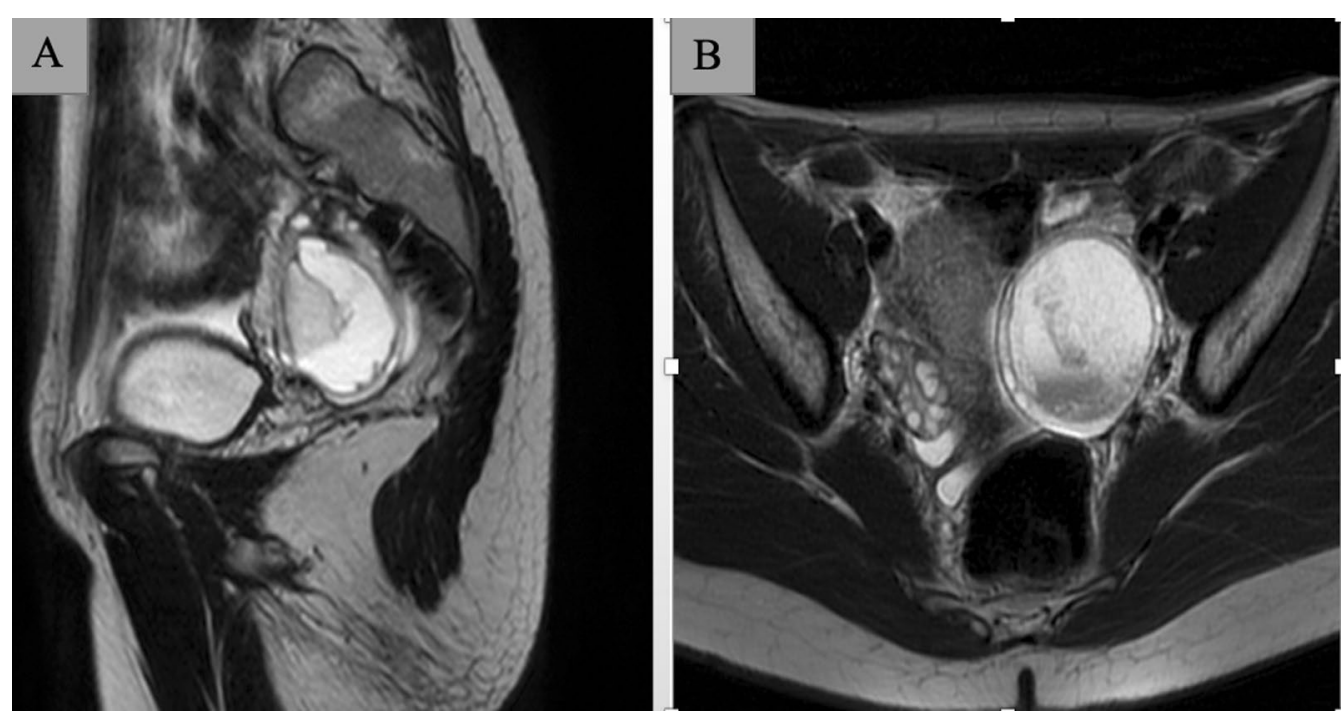

Fig. 4 Sagital (A) and axial (B) T2 weighted MR image shows high signal intensity on T2WI with blood-fluid level (the hypointense sediment indicates blood products), and hypointense wall

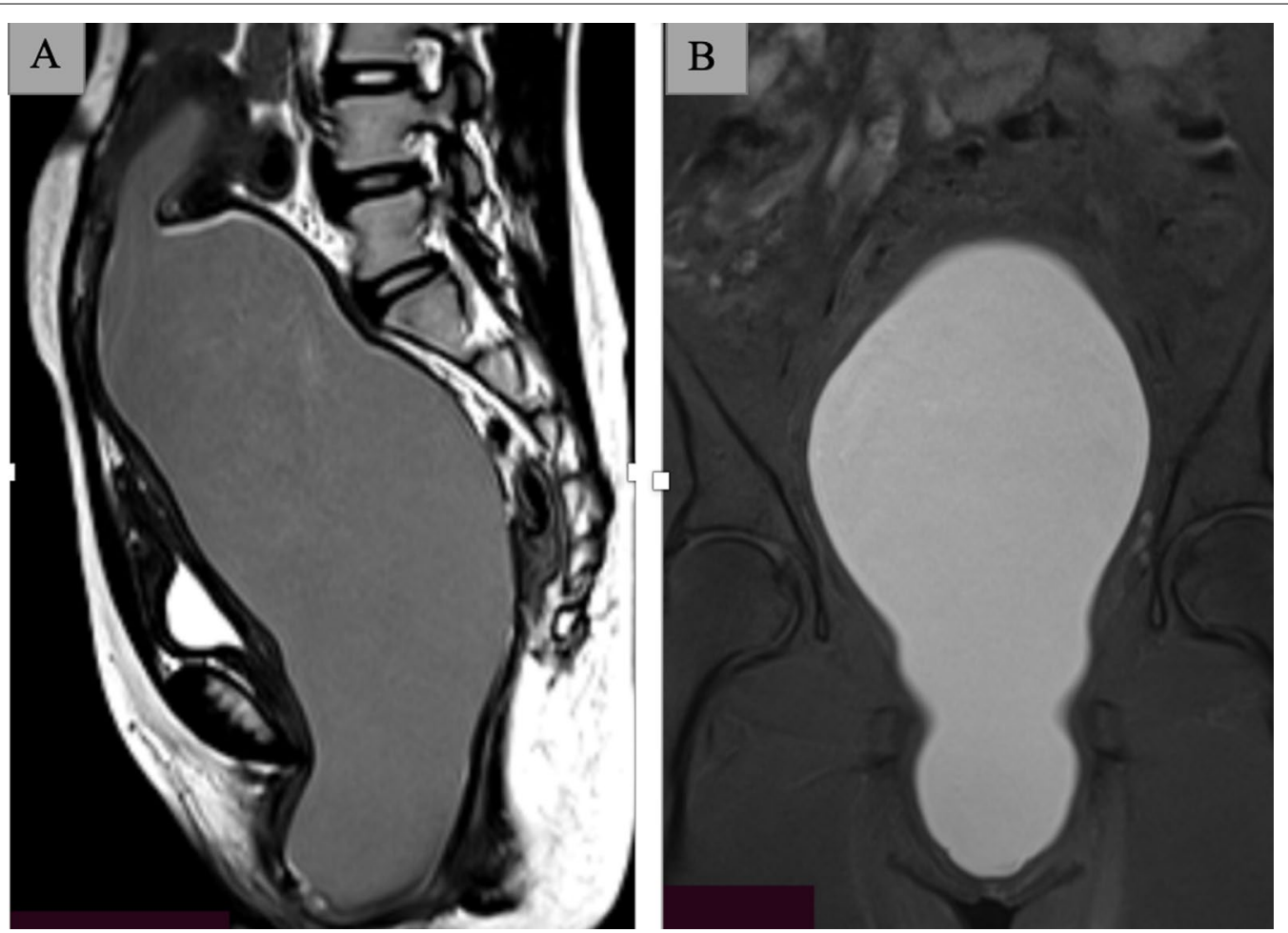

Fig. 5 T2 weighted sagital (A) and T1 weighted coronal (B) images showing uterine cavity filled with blood which appears hypointense on T2 and hyperintense on $\mathrm{T1}$ weighted image-suggesting hematometra

appearing hypointense on T1W images (B) and displaying restricted diffusion on DW images $(\mathrm{C})$ with low ADC values on $\mathrm{ADC} \operatorname{map}(\mathrm{D})$.
2 boys presented with the acute onset pain in scrotal region and testicular torsion was suspected in those patients. 1 patient had MRI findings of testicular torsion. 


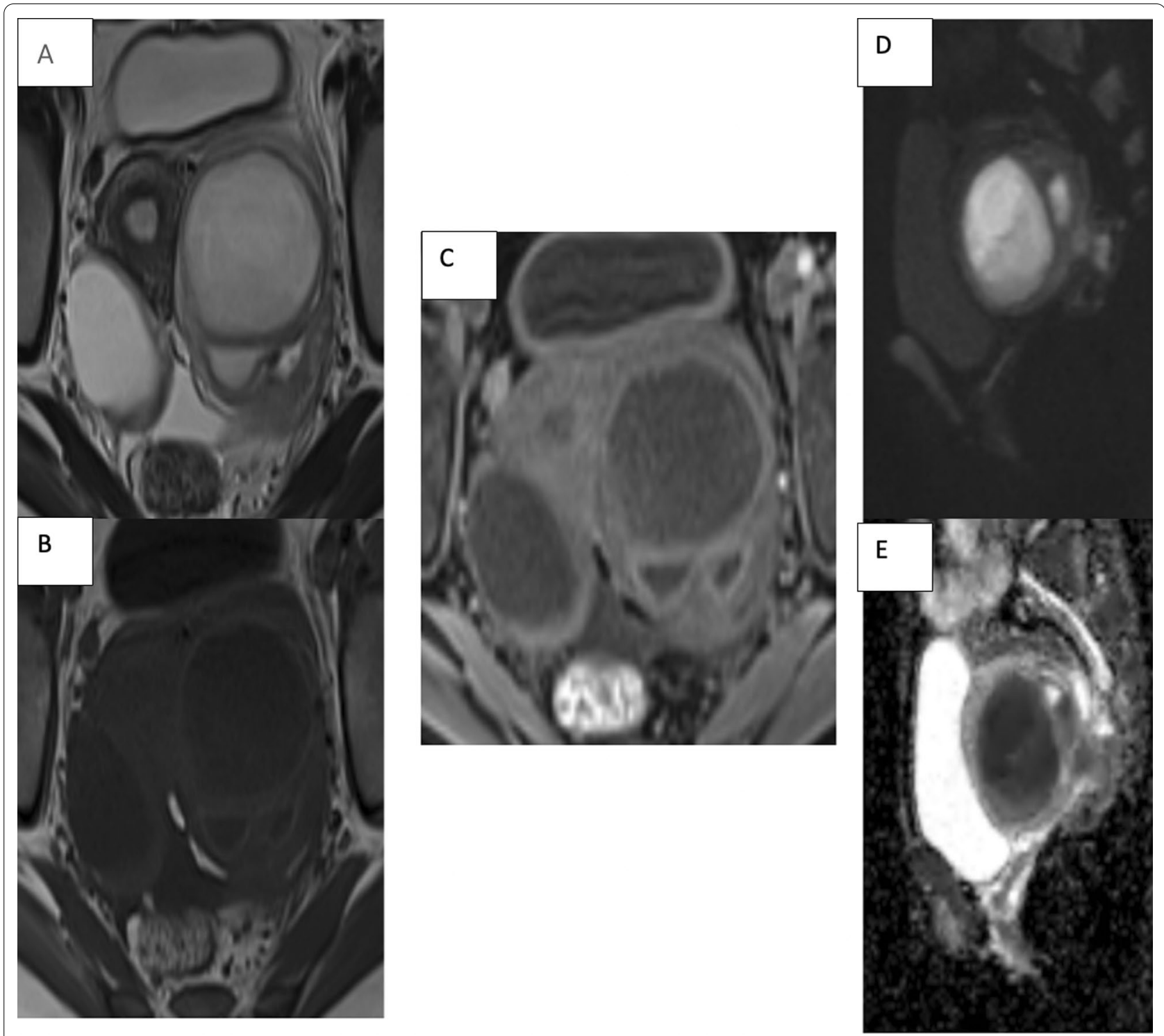

Fig. 6 Tubo-ovarian abscess. The axial T2WI (A) in this patient showed encapsulated hyperintense contents, appearing hypointense on T1W images (B) and displaying restricted diffusion on DW images (C) with low ADC values on ADC map (D)

Testicular torsion requires emergency surgical treatment because any delay in surgical intervention may result in irreversible testicular damage due to impaired testicular blood flow [17]. MRI findings in patient were low signal intensities with spotty or streaky patterns in fat-suppressed T2-weighted (Fig. 7). The testicular torsion was proved after surgery.

1 patient after having acute abdominal pain was diagnosed with meckel's diverticulum. He underwent MRI. No significant findings were suggested on MRI, but proved to be meckel's diverticulum after surgery on histopathology.
Although uncommon in young children, acute cholecystitis is another cause of abdominal pain that requires surgical consultation. Acute cholecystitis is often caused by an impacted gallstone at the gallbladder neck or within the cystic duct. The MRI findings of acute cholecystitis in present study included distended fluid-filled gallbladder with gallstones, gallbladder wall thickening, gallbladder wall edema, and pericholecystic fluid. Gallstones and gallbladder wall thickening were best seen on fat sat $\mathrm{T} 2$. Pericholecystic fluid and gall-bladder wall edema were best seen on fat-suppressed single-shot T2-weighted sequence. Loud et al. [18] described the similar findings of acute cholecystitis on MRI in pediatric age group. 


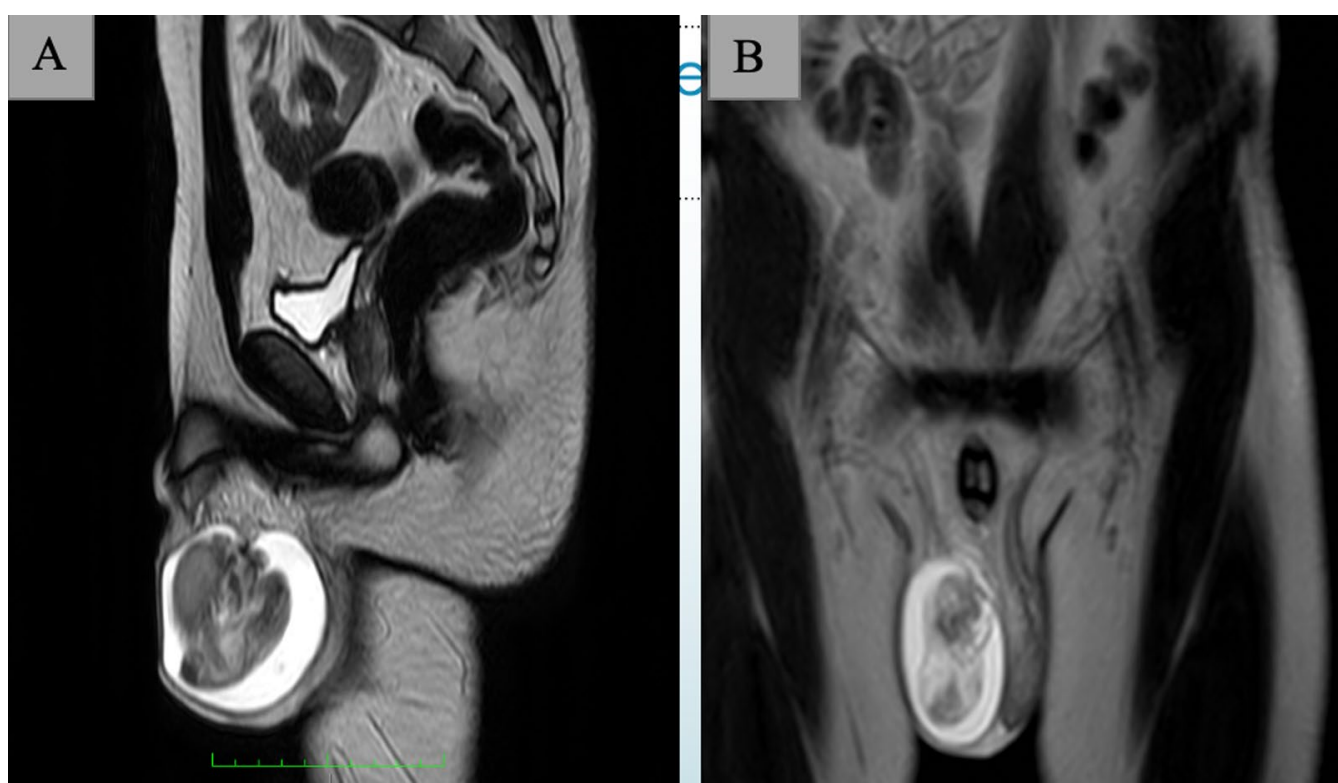

Fig. 7 T2 weighted sagital (A) and coronal (B) images showing enlarged right testis with isointense signal and spotty pattern

MRI provides rapid, accurate identification of smallbowel obstruction and assists in the determination of cause without exposing the patient to radiation. MRI also utilizes intraluminal air as a natural contrast agent and is not limited by previous administration of barium. The diagnosis of small-bowel obstruction on MRI is similar to $\mathrm{CT}$ and involves identifying dilated loops of bowel proximal to the obstruction, a distinct transition point, and normal caliber or collapsed bowel distally (Fig. 8). Multiplanar capabilities of MRI allow visualization of the cause of small-bowel obstruction. Rapid scanning with MRI using the HASTE sequence can, within seconds, evaluate smallbowel obstruction with a high degree of accuracy [19].

Pancreatitis is a common pediatric presentation and its prevalence is increasing. There is significant morbidity associated with this condition. In adults, CT is considered the most appropriate staging modality for acute pancreatitis with or without necrosis; however, MRI is increasingly
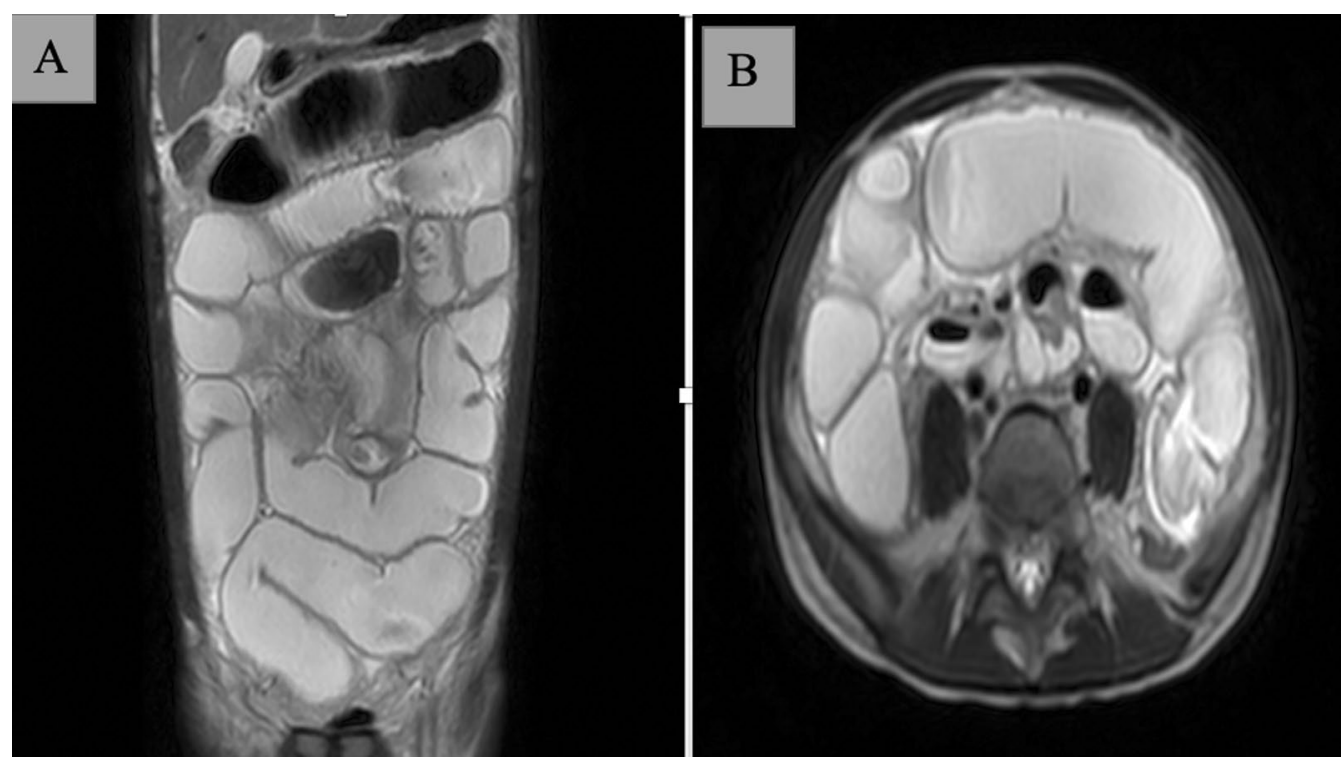

Fig. 8 Rapid haste T2 weighted coronal (A) and axial (B) images showing dilated loops of large bowel 


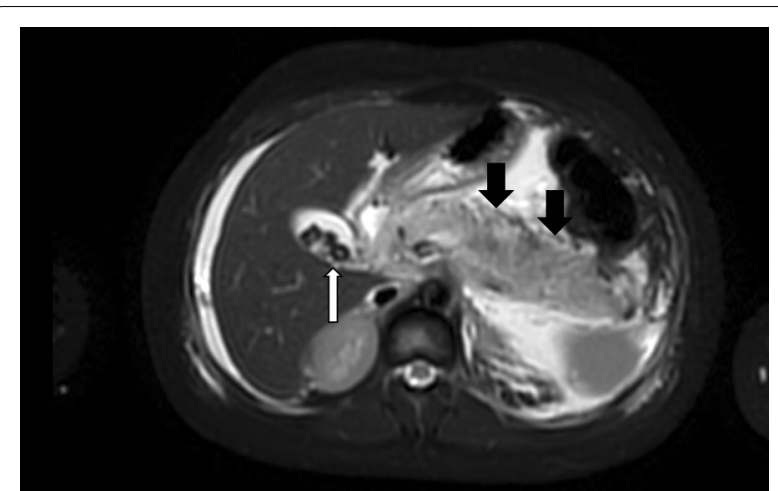

Fig. 9 T2 weighted FS axial image showing enlarged pancreas with diffuse hyperintesity along with moderate amount of peripancreatic fluid (black arrows) associated with multiple calculi seen in visualised gall bladder as hypointense foci which is the cause for pancreatitis in this patient (white arrow)

preferred for the pediatric population [20]. The imaging findings on MRI in our study were pancreatic enlargement with loss of the usual lobular appearance of the pancreatic margins resulting in a sausage like appearance and subtle parenchymal signal changes of relative T2 hyperintensity and T1 hypointensity with mild diffusion restriction suggesting the diagnosis (Fig. 9). Jae-Yeon Hwang et al. [21] described the imaging features in pediatric patients having pancreatitis. They found that the signal intensity of the inflamed pancreas is high on T2-weighted images, but remains normal or slightly low on T1weighted fatsuppressed images depending on the severity of pancreatic inflammation.

Acute urinary tract obstruction caused by urolithiasis is frequently seen in adults, although recent studies have shown an increased prevalence even in the pediatric age group. Most children present with acute flank pain that may be accompanied by hematuria. Typical locations of obstructive stones in the urinary tract include the ureteropelvic junction, the ureter at the level of the pelvic brim, and the ureterovesical junction. Management of obstructive urolithiasis is based on clinical features, the size and location of the stones, stone composition, and renal function. The obstructive stone itself often is not identified on MRI because most stones are small and have low signal on all sequences [21]. In our study, we were able to visualise the exquisite details of ureteric dilation, periureteric edema, dilated renal pelvis and calyces, renal edema, and presence of perinephric edema due to high contrast resolution with coronal and axial T2-weighted sequences. Chung et al. [22] also described similar findings on MRI in acute urinary tract conditions. Another condition which can cause acute urinary obstruction is pelvi-ureteric junction obstruction (Fig. 10).
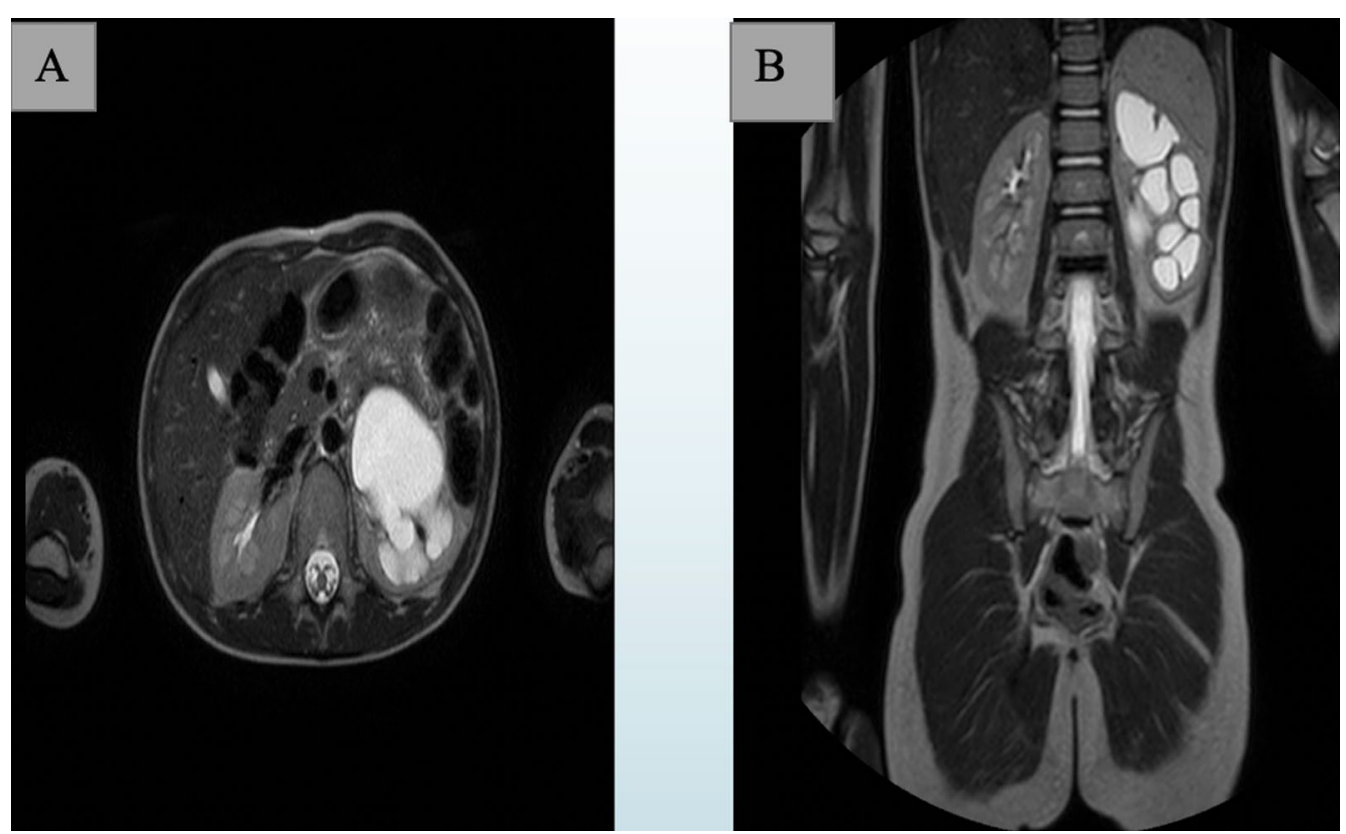

Fig. 10 Haste T2 weighted axial (A) and coronal (B) images showing pelviureteric obstruction with moderate hydronephrosis of left kidney not associated with dilatation of ipsilateral ureter suggesting the pelviureteric obstruction 


\section{Conclusion}

Unenhanced MRI is an excellent option for the initial, detailed evaluation of acute abdominal emergencies in pediatric patients because it can diagnose the whole range of presenting abnormalities which include the causes of abdominal pain warranting surgical and nonsurgical management. MRI could serve an important role as an alternative to $\mathrm{CT}$ when ultrasound findings are equivocal and to lessen the exposure of pediatric patients to ionizing radiation.

\section{Limitation}

Limitations of MR imaging included poorer spatial resolution compared with that of $\mathrm{CT}$, increased sensitivity to motion-related artifacts, and limited compatibility with equipment used in intensive care and monitoring of patients. The small sample size in our study is also one of the limitation.

\begin{abstract}
Abbreviations
MRI: Magnetic resonance imaging; CT: Computed tomography; HASTE: Half Fourier Acquisition Single-shot Turbo spin Echo; MRCP: Magnetic resonance cholangiopancreatography; SPAIR: Spectral Attenuated Inversion Recovery; DWI: Diffusion weighted imaging; FOV: Field of view; TE: Time to Echo; TR: Repetition time.
\end{abstract}

\section{Acknowledgements}

None.

\section{Authors' contributions}

Dr. VA-Conceptualization, methodology, validation, formal analysis, writingoriginal draft, review and and editing, visualization, supervision and project administration. Dr. TK-Methodology, formal analysis, writing-original draft and visualization. Dr KS - Conceptualization, methodology, validation and supervision. All the authors have read and approved the submitted version and have agreed both to be personally accountable for their contributions and to ensure that the questions related to the accuracy or integrity of any part of the work are appropriately investigated and resolved. All authors read and approved the final manuscript.

\section{Funding}

This research did not receive any specific grant from funding agencies in the public, commercial, or not-for-profit sectors.

\section{Declarations}

\section{Ethics approval and consent to participate}

This study is in accordance with the ethical standards of Institutional Review Board and the Declaration of Helsinki. The full name of the IRB/IRC Issuing committee: Research and ethical committee SGRDIMSAR, Amritsar. The reference number of IRB/IRC approval: SGRDU/cont/thesis/20-92. All patients which were included in this research, their parents or legal guardians gave written informed consent to publish the data contained within this study.

\section{Consent for publication}

\section{Yes.}

\section{Competing interests}

The authors declare that they have no competing interests.

\section{References}

1. Reust CE, Williams A (2016) Acute abdominal pain in children. Am Fam Physician 93(10):830-836

2. Horner KB, Jones A, Wang L, Winger DG, Marin JR (2016) Variation in advanced imaging for pediatric patients with abdominal pain discharged from the ED. Am J Emerg Med 34:2320-2325

3. Koberlein GC, Trout AT, Rigsby CK, et al.; Expert Panel on Pediatric Imaging (2019) ACR appropriateness criteria ${ }^{\circledR}$ suspected appendicitis: child. J Am Coll Radiol 16(5S):S252-S263

4. Binkovitz LA, Unsdorfer KML, Thapa P et al (2015) Pediatric appendiceal ultrasound: accuracy, determinacy and clinical outcomes. Pediatr Radiol 45:1934-1944

5. Birnbaum BA, Jeffrey RB Jr (1998) CT and sonographic evaluation of acute right lower quadrant pain. AJR 170:361-371

6. Brenner DJ, Elliston CD (2004) Estimated radiation risks potentially associated with full-body CT screening. Radiology 232:735-738

7. Mushtaq R, Desoky SM, Morello F et al (2019) First-line diagnostic evaluation with MRI of children suspected of having acute appendicitis. Radiology 291:170-177

8. Moore MM, Gustas CN, Choudhary AK et al (2012) MRI for clinically suspected pediatric appendicitis: an implemented program. Pediatr Radiol 42:1056-1063

9. Duke $\mathrm{E}$, Kalb B, Arif-Tiwari H et al (2016) A systematic review and meta-analysis of diagnostic performance of MRI for evaluation of acute appendicitis. AJR 206:508-517

10. Kim JR, Suh $\mathrm{CH}$, Yoon HM et al (2018) Performance of MRI for suspected appendicitis in pediatric patients and negative appendectomy rate: a systematic review and meta-analysis. J Magn Reson Imaging 47:767-778

11. Puylaert JB (2003) Ultrasonography of the acute abdomen: gastrointestinal conditions. Radiol Clin N Am 41:1227-1242

12. Choudhary AK, Donnelly LF, Racadino JM, Strife JL (2007) Diseases associated with childhood obesity. AJR 188:1118-1130

13. Ionising Radiation (Medical Exposure) Regulations 2000 (SI 2000 No 1059), London, HMSO, http://www.opsi.gov.uk/si/si2000/20001059.htm

14. Johnson A, Filippi C, Andrews T, Higgins T, Tam J, Keating D et al (2012) Ultrafast 3-T MRI in the evaluation of children with acute lower abdominal pain for the detection of appendicitis. Am J Roentgenol 198(6):1424-1430

15. Hibbard LT (1985) Adnexal torsion. Am J Obstet Gynecol 152:456-461

16. Sintim-Damoa A, Majmudar AS, Cohen HL, Parvey LS (2017) Pediatric ovarian torsion: spectrum of imaging findings. Radiographics 37:1892-1908

17. Anderson JB, Williamson RCN (1986) The fate of the human testes following unilateral torsion of the spermatic cord. Br J Urol 58:698-704

18. Loud PA, Semelka RC, Kettritz U, Brown JJ, Reinhold C (1996) MRI of acute cholecystitis: comparison with the normal gallbladder and other entities. Magn Reson Imaging 14:349-355

19. Regan F, Beall DP, Bohlman ME, Khazan R, Sufi A, Schaefer DC (1998) Fast MR imaging and the detection of small-bowel obstruction. AJR 170:1465-1469

20. Thai TC, Riherd DM, Rust KR (2013) MRI manifestations of pancreatic disease, especially pancreatitis, in the pediatric population. AJR 201:W877-W892

21. Hwang J, Yoon H, Kim K. Characteristics of pediatric pancreatitis on magnetic resonance cholangiopancreatography. Pediatr Gastroenterol Hepatol Nutr. 2015;18(2):73.

22. Chung AD, Schieda N, Shanbhogue AK, Dilauro M, Rosenkrantz AB, Siegelman ES. MRI evaluation of the urothelial tract: pitfalls and solutions. AJR. 2016;207:W108-W116. https://doi.org/10.2214/AJR.16.16348.

\section{Publisher's Note}

Springer Nature remains neutral with regard to jurisdictional claims in published maps and institutional affiliations. 